\title{
NOVO FINANCIAMENTO DA ATENÇÃO PRIMÁRIA NO BRASIL: O QUE MUDOU E POSSIIVEIS CONSEQUÊNCIAS
}

\section{NEW FINANCE FOR PRIMARY CARE IN BRAZIL: WHAT HAS CHANGED AND WHICH ARE THE POSSIBLE CONSEQUENCES}

\author{
Mateus Louis Rodrigues Cavalcante ${ }^{1}$ \\ Ana Flávia Henriques Ribeiro Monteiro ${ }^{2}$ \\ Ana Paula Monteiro do Nascimento ${ }^{3}$ \\ Lucas Galvão Araújo ${ }^{4}$ \\ Paloma Medeiros Gomes Cavalcanti ${ }^{5}$ \\ Raissa Cavalcanti Rodrigues ${ }^{6}$
}

\begin{abstract}
RESUMO: OBJETIVO: Discorrer sobre a mudança proposta através do novo modelo de financiamento da Atenção Primária brasileira chamado de Previne Brasil. MÉTODO: Utilizou-se a base de dados da Associação Brasileira de Saúde Coletiva (ABRASCO), Organização Mundial de Saúde e Portarias oficiais do Governo brasileiro, além de artigos encontrados no PUBMED e Google Acadêmico para estudo e correlação com os dados pesquisados. RESULTADOS: Foi-se observado após a análise dos documentos consultados que 0 a proposta de novo financiamento para a atenção primária a saúde do Brasil foi um projeto pouco debatido nas Conferências Nacionais de Saúde e com os órgãos de representatividade social brasileiros. Além disso, o novo programa de financiamento, chamado de Previne Brasil, muda a forma de repasse de recursos financeiros sem oferecer meios para que os municípios consigam aderir a nova medida, dificultando, então sua efetividade. CONCLUSÃO: O Previne Brasil precisa ser melhor debatido com as instâncias representativas sociais brasileiras como determina a Constituição Federal e a Lei 8080/90. Além disso, precisa-se de caminhos mais claros para colocar o projeto em prática para os profissionais e gestores dos municípios.
\end{abstract}

Palavras chave: Atenção Primária. Financiamento SUS. Saúde Pública.

\footnotetext{
${ }^{1}$ Estudante de Medicina do $11^{\circ}$ período do Centro Universitário de João Pessoa - UNIPE.

${ }^{2}$ Estudante de Medicina do $8^{\circ}$ período do Centro Universitário de João Pessoa - UNIPE.

${ }^{3}$ Estudante de Medicina do $8^{\circ}$ período do Centro Universitário de João Pessoa - UNIPE.

${ }^{4}$ Estudante de Medicina do $8^{\circ}$ período do Centro Universitário de João Pessoa - UNIPE.

${ }^{5}$ Estudante de Medicina do $8^{\circ}$ período do Centro Universitário de João Pessoa - UNIPE.

${ }^{6}$ Estudante de Medicina do $5^{\circ}$ período do Centro Universitário de João Pessoa - UNIPE.
} 
ABSTRACT: OBJECTIVE: To discuss the proposed change through the new model called Previne Brasil for financing Brazilian Primary Care. METHOD: The database of the Brazilian Association of Collective Health (ABRASCO), the World Health Organization and official ordinances of the Brazilian Government were used, in addition to articles found in PUBMED and Google Scholar for study and correlation with the researched data. RESULTS: It was observed after analyzing the documents consulted that the proposal for new financing for primary health care in Brazil was a project that was little debated at the National Health Conferences and with the Brazilian social representative bodies. In addition, the new financing program, called Previne Brasil, changes the way of transferring financial resources without offering the means for municipalities to adhere to the new measure, thus hampering its effectiveness. CONCLUSION: Previne Brasil needs to be better discussed with the brazilian social representative bodies as determined by the federal constitution and at the Law 8080/90. Besides that, clearer paths are needed to put the project into practice for professionals and managers in the municipalities.

Keywords: Primary Health Care. SUS financing. Public health. 


\section{INTRODUÇÃO}

Para que se possa entender como financiar um sistema de saúde é fundamental saber como esses sistemas funcionam, quais são seus princípios e o que se pretende fazer a partir do compromisso estabelecido pelo programa optado pelo governo. Sabidamente, no mundo existem vários sistemas de saúde, cada um com suas características peculiares (ROEMER, 1994).

Sendo assim, o financiamento da saúde ocorre de maneiras diferentes nos mais de 200 países do mundo. Cada nação tem sua própria forma de viabilizar o acesso à saúde para o seu povo. A opção de adequar o modo de financiamento a saúde de acordo como as diversas realidades é natural uma vez que cada nação tem seus costumes, cultura e modo de interagir com o seu contexto social e histórico. Apesar disso, análises de vários sistemas de saúde feita por uma associação chamada de Physicians For National Health Program (PNHP) propõe grupos de como funcionam os sistemas de saúde dos países, visando conhecer as características de cada programa para conseguir entende-lo (PNHP, 2020). 
Percentage of population covered by health insurance, 2011

Estimate of health insurance coverage as a percentage of total population. Coverage includes affiliated members of health insurance or estimation of the population having free access to health care services provided by the State.

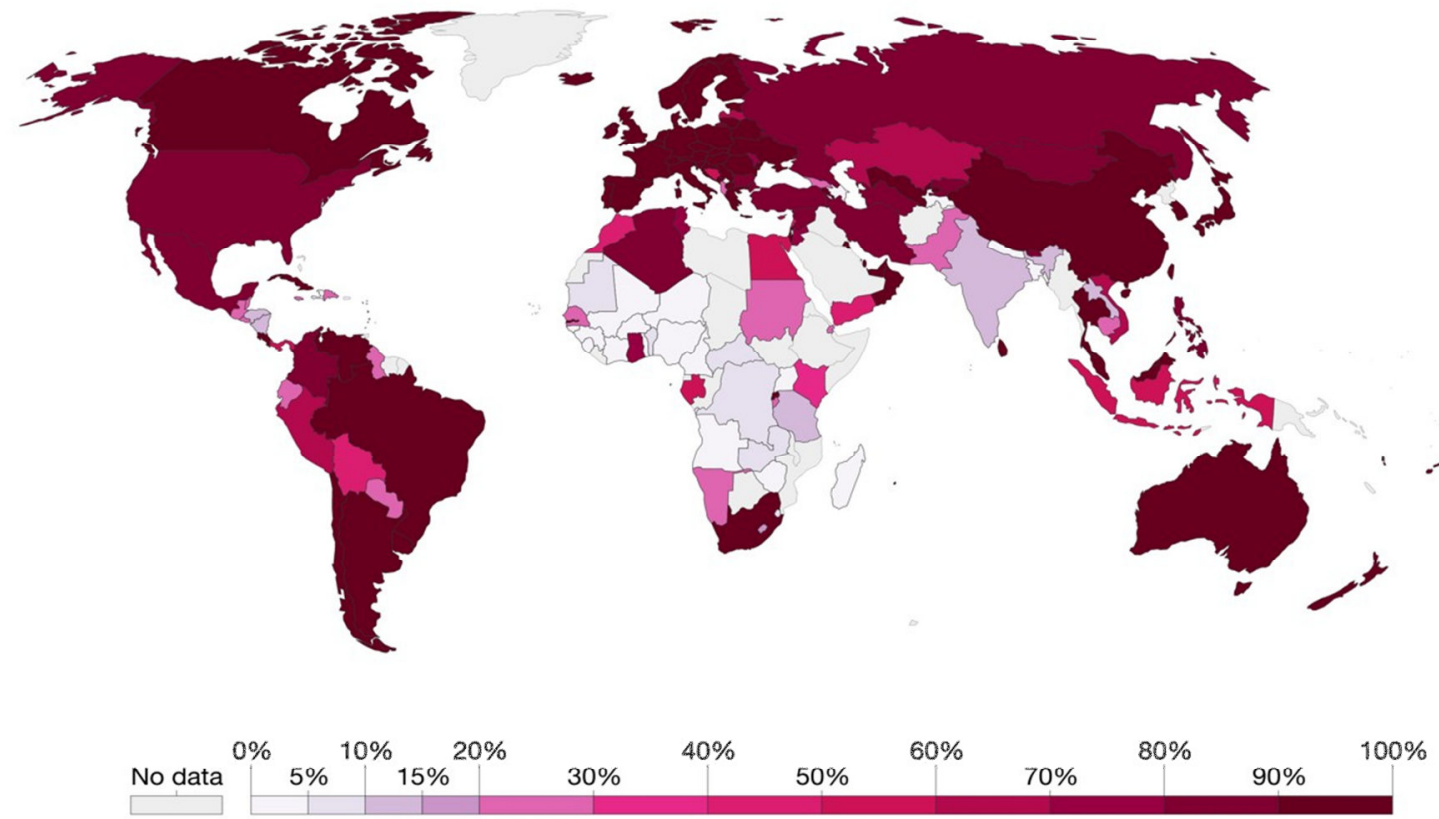

Imagem 1: Porcentagem da população de cada país coberta por algum sistema de saúde em 2011. Fonte: Our WorldInData

As diversas nações do mundo têm interesse, naturalmente, em oferecer o básico em saúde para sua população. Isso significa ofertar dignidade e além disso, vendo por uma perspectiva econômica e desenvolvimentista para um país, oferecer saúde também é viabilizar o progresso de uma nação, dar condições para que pessoas produzam para os seus países e melhorem a vida dos demais cidadãos, gerem mais renda e riqueza para o seu povo (OMS, 2010).

Sobre os modelos de sistema de saúde, o financiamento acontece, de maneira geral e simplificada, de 4 formas diferentes. A primeira é o financiamento através do setor privado, chamado em inglês de out-of-pocket, por meio de empresas de seguros de saúde oferecendo serviços que são aderidos pelos consumidores. O segundo modo é por meio de promoção de saúde pública, também conhecido como Beveridge model, onde o estado é o responsável pelo financiamento da saúde de sua população, criando políticas e programas que 
viabilizem o acesso aos serviços para a população que precisa. O terceiro modelo é uma mistura dos dois anteriores onde o setor privado e o público se mesclam para oferecer serviços para a população, é um modelo onde o pagamento pelos serviços vem do setor público. Nesse modelo pode haver hospitais privados que prestam serviço à população e tem seu pagamento feito pelo governo. Há, por fim, um quarto modelo que financia o sistema de saúde com fundos de saúde através de rendas provindas de múltiplas fontes, caindo em um fundo único que não visa o lucro. Esse é regulado e controlado pelo governo para oferecer recursos financeiros aos serviços, é também chamado de Bismark model (PNHP, 2020).

O primeiro modelo, tem como premissa liberalista que o cidadão é livre para fazer suas escolhas e as consequências acarretadas por elas. É, assim, um modelo presente em países onde tem o liberalismo econômico no plano regente da sua política governamental ou em países onde não há recursos financeiros e organização suficientes para se estruturar um sistema de saúde.

Dessa forma, o principal exemplo do primeiro modelo é os Estados Unidos da América (EUA). A opção de aderir a esse modo de saúde isenta o estado da responsabilidade de oferecer saúde, consequentemente seus cidadãos não pagam tributos destinados a esse serviço, já que o estado não o oferece. Essa situação, portanto, deixa as pessoas livres para escolherem os serviços que mais se adequam às suas realidades. Isso também significa que quem não tem recursos para contratar um serviço está excluído de receber saúde e se precisar, terá que pagar posteriormente pelos serviços prestados, acarretando dívidas (PNHP, 2020).

Esse modelo é também o modelo da maioria dos países, principalmente os mais pobres, que não têm a organização necessária nem os fundos para estabelecerem um sistema de saúde. Estima-se que somente 40 dos mais de 200 países do mundo tenham sistema de saúde, para o restante das pessoas somente tem-se acesso aos médicos ou aos serviços de saúde caso se tenha dinheiro para pagar pelo serviço. Para os EUA há um paradoxo, pois embora esse seja o país mais rico do mundo, para aproximadamente $15 \%$ da sua população a realidade é semelhante a da Camboja ou Índia rural, onde só se tem acesso à saúde quando se tem dinheiro (PNHP, 2020). 
O segundo modelo parte de uma premissa de que o estado é o provedor do bem-estar social e das condições que viabilizem o desenvolvimento da nação (PNHP, 2020), oferecendo saúde para receber progresso e desenvolvimento (OMS, 2010). Dessa forma, está mais presente em países pobres e em desenvolvimento, é também conhecido como acesso universal à saúde. O país que opta por oferecer acesso à saúde a todos os cidadãos recebe a contribuição por meio de impostos e cria políticas e programas para serem seguidos pelos gestores públicos. Além disso é marcante nesse tipo de sistema que os hospitais sejam públicos e até os profissionais sejam funcionários contratados pelo governo. Os principais representantes desse grupo são: Cuba e Reino Unido (PNHP, 2020).

O terceiro modo de oferecer saúde é uma junção dos dois modelos anteriores, onde os cidadãos têm a sua disposição os dois tipos de modelo um privado e um público e podem usar qualquer um deles. Existe, nesse contexto, diversas formas de funcionamento; desde um sistema de saúde público com outro suplementar, como acontece em alguns países, como Espanha, até sistemas onde os dois modelos coexistem com o setor privado recebendo recursos públicos para prestarem serviço a população sem custo diretamente ao consumidor, como acontece no Brasil. Essa situação é observada quando hospitais e clínicas realizam exames e outros serviços para os dois setores, público e privado (ROEMER, 1994).

O quarto modelo tem o seu uso principalmente em países da Europa. Ele funciona a partir do recebimento de renda de vários setores para, no fim, criar um fundo único que é administrado pelos gestores do estado. Os países que usam esse tipo de sistema de financiamento são: Alemanha, França, Bélgica e Holanda. 


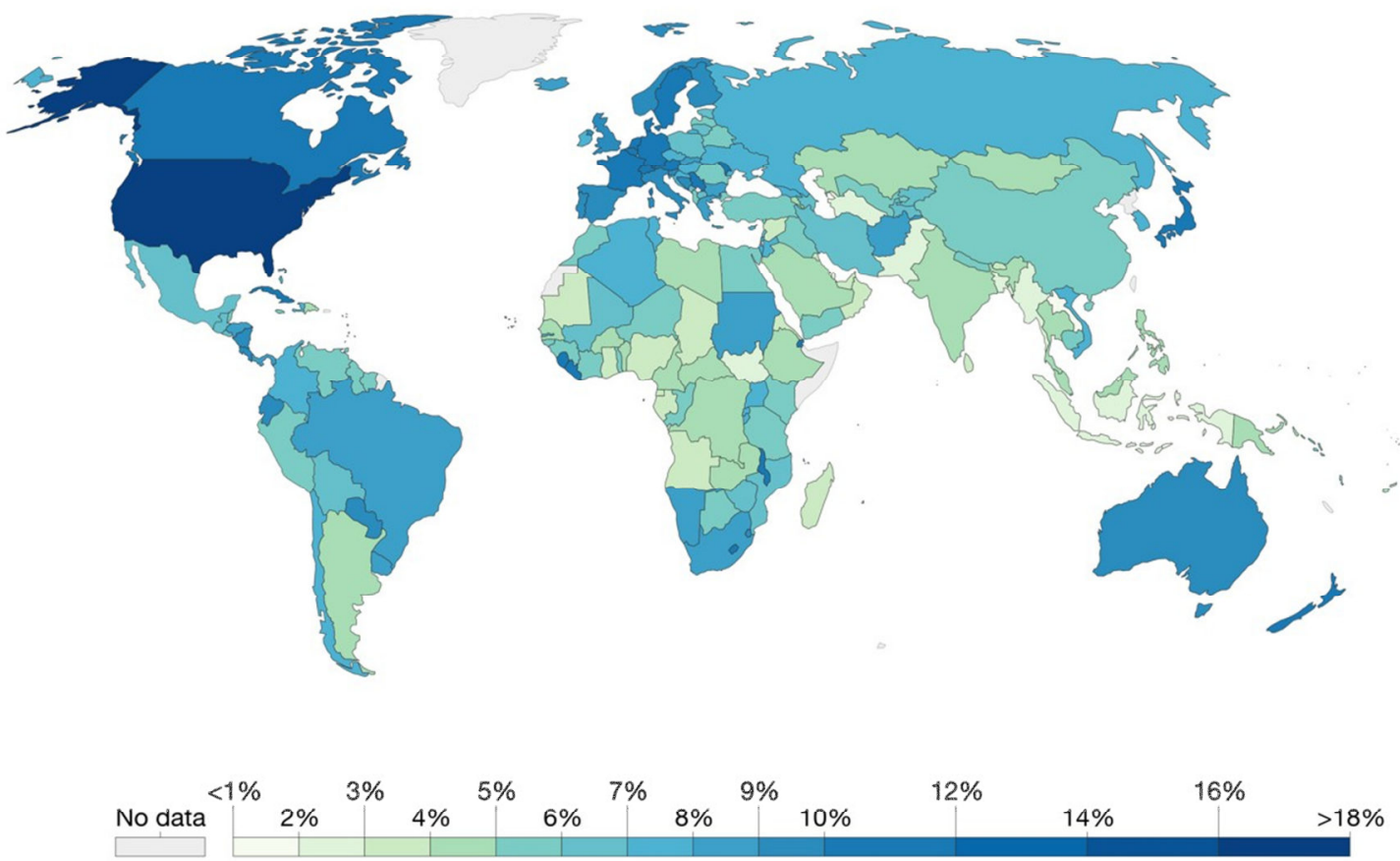

Imagem 2: Custeio do sistema de saúde segundo a porcentagem do produto interno bruto no mundo. Fonte: Our WorldlnData.

Para que todos esses sistemas de saúde acima citados existam é preciso, naturalmente, que exista financiamento para viabilização de consultas, exames, medicamentos e as demais formas de acesso ao cuidado. Internacionalmente, investe-se de 5 a $12 \%$ do produto interno bruto (PIB) para financiar os sistemas de saúde (ORTIZ-OSPINA; ROSER, 2020). No Brasil, investe-se aproximadamente 6\% do PIB. No mundo, de acordo com a OMS (2010), um investimento de $60 \$$ per capta (60 dólares internacionais para cada pessoa) é o valor necessário para se ter um sistema de saúde universal para que possa atender a população, no Brasil o valor praticado atualmente é o de aproximadamente $7 \$$ ou 23 a 28 reais, que é o valor da PAB fixa (BRASIL, 2017). Há dados do Banco Mundial de 2014 explicitando que o Brasil investe aproximadamente $8 \%$ do seu produto interno bruto em saúde (representado no gráfico acima). 
A Constituição Federal brasileira de 1988 no artigo n 196 afirma que a saúde é um direito de todos e dever do estado (BRASIL, 1988). Para que isso acontecesse, foi proposto um programa em 1990 através da lei 8080 fundando o Sistema Único de Saúde (SUS). A lei 8090/1990 também dá as características de operacionalização do SUS, falando sobre o financiamento do sistema, com um mínimo de recursos provenientes da união, dos estados e dos municípios com $6 \%$ até $7 \%, 12 \%$ e $15 \%$ respectivamente dos recursos arrecadados (BRASIL,1990). Esse montante é usado para financiar unidades básicas de saúde e suas equipes, os hospitais públicos, incluindo os universitários, os laboratórios e hemocentros (bancos de sangue), os serviços de vigilância sanitária, vigilância epidemiológica, vigilância ambiental, além de fundações e institutos de pesquisa acadêmica e científica, como Fundação Oswaldo Cruz e o Instituto Vital Brasil. Além disso, comprar todos os insumos necessários para esses serviços.

Diante do exposto, sabendo que o Brasil se propõe a oferecer saúde integral para os seus habitantes, o valor praticado no momento inviabiliza que aconteça tal propósito em todos os níveis de saúde, dos mais básicos até os mais complexos e sofisticados uma vez que o recurso é insuficiente para praticar saúde universal, situação essa que vem a décadas sendo discutida como "subfinanciamento do SUS".

Nesse contexto, houve um novo projeto que muda o modo de financiamento da atenção primária do Sistema de Saúde brasileiro alterando a forma de repasse para esse setor da saúde. Sabendo disso, esse trabalho se propõe a analisar a nova proposta e discorrer sobre a mudança proposta através do novo modelo chamado de Previne Brasil de financiamento da Atenção Primária brasileiro falando brevemente sobre o que é o novo sistema, os prazos dados até que mudança ocorra. 


\section{MÉTODO}

Trata-se de um estudo de revisão bibliográfica, retrospectivo, realizado por meio da consulta ao Portal do Ministério da Saúde e em bases oficiais do Conselho Nacional de Secretarias Municipais de Saúde e O Conselho Nacional de Secretários de Saúde assim como em bases de dados de associações de saúde do Brasil, como Associação Brasileira de Saúde Coletiva (ABRASCO) e na base de dados do site da Organização Mundial de Saúde (OMS). Além disso, houve pesquisa bibliográfica em artigos para estudo e correlação com os dados pesquisados. Tais artigos foram pesquisados nas bases de dados PUBMED e Google Acadêmico.

\section{DISCUSSÃO}

Em 12 de novembro de 2019 o governo brasileiro lança a portaria $n^{\circ} 2.979$, essa institui um novo projeto chamado de "Previne Brasil". Esse programa muda como os recursos serão repassados a partir de 2021 para a Atenção Primária no Brasil. O financiamento desse setor da saúde, antes dessa portaria, acontecia através do Piso de Atenção Básica (PAB), sendo um valor fixo per capta do município e outro valor variável de acordo com os programas aderidos e os resultados obtidos. Com o Previne Brasil, propõe-se que o pagamento a partir de 2021 aconteça de acordo com a quantidade de usuários cadastrado por equipes de saúde até abril 2020 (BRASIL, 2019a).

Entendendo a formação desse programa, a proposta foi pouco discutida com os setores sociais, isso por que se tem a citação da aprovação do novo modelo de financiamento uma única da Comissão de Intergestores Tripartite (CIT) de 29 de outubro de 2019 (HARZHEIM, 2020). É muito incomum que se tenha entrado em consenso para a aprovação de uma reformulação do financiamento de toda a rede 
de Atenção Primária em uma única reunião. Após essa reunião, passaram-se somente 13 dias e o novo programa foi lançado.

Dar-se, dessa forma, a impressão que a proposta foi imposta porque uma vez que essa proposta não foi um pleito levantado pelas Conferências Nacionais de Saúde (CNS) e também não teve tempo necessário para ser discutida, não foi então debatida e formulada em conjunto com os setores sociais constituintes do SUS, como é o regimento constitucional presente da Lei Orgânica 8080/90 de formação do SUS. Sendo assim, não se foi respeitado o rito para a formação de novas políticas para a saúde. Esse ambiente poderá ocasionar alguns problemas, como a surpresa dos profissionais e gestores e a incapacidade de se adequar tão rapidamente com o novo sistema para se conseguir viabiliza-lo (BRASIL, 2019b).

Além disso, o programa também exige que se realize o cadastro do usuário por equipes de saúde já com muitas tarefadas e sobrecarregadas de trabalho. Mesmo sabendo dessa situação, não são oferecidos recursos e não contrata-se novos profissionais. Deseja-se que pessoas sejam cadastradas as suas equipes, ficando o questionamento se os profissionais terão que parar o atendimento às pessoas para focarem no cadastro dos usuários e assim não ficarem sem recursos para o futuro. Sendo assim, essa situação imaginativa é ilusória e sem precedentes no mundo real. Foi-se pensando em um programa, mas não se pensou na sua execução. No programa expõe-se apenas aconselhamentos genéricos de como se conseguir o cadastro, porém esses não são suficientes para se adequarem ao mundo real (ABRASCO, 2019).

A maioria das equipes de saúde deseja e se empenha para conseguir conhecer o seu território, isso por que é satisfatório para a equipe conseguir se programar para cuidar da população circunscrita. Além disso, já é proposto pelo regimento atual do SUS o cadastro dos usuários. Isso não acontece por fatores como alta carga de atendimento de usuários todos os dias pelas equipes, falta de apoio do município para o cadastro das pessoas. O cadastro almejado no Previne Brasil é o de 50 milhões de novas pessoas, pelas as mesmas equipe e com as mesmas condições encontradas hoje (BRASIL, 2019a).

Uma outra realidade encontrada é citada por Cavalcanti et al. no atual sistema é a falta de organização de muitas equipes em todo o brasil, com falta de 
planejamento dos gestores, sem agentes comunitários de saúde (ACS) suficientes, sem dados suficientes. Além disso, tem- se, em muitos casos, falta de equipamentos para os ACS e o despreparo deles para fazerem o cadastro das pessoas descobertas. Mesmo sabendo disso, dar-se o prazo até abril para o cadastro dessas pessoas.

Exemplificando essa situação dos ACS, pode-se perceber algumas discrepâncias entre as equipes de saúde, algumas equipes têm tablets onde conseguem fazer o cadastro e registrar no momento os usuários; outras equipes, grande parte delas em municípios mais pobres e afastados dos grandes centros, têm somente caneta e papel e fazem o cadastro para depois enviarem o relatório para a secretaria do município registrar o cadastro. Essa última situação precisa de uma logística maior e dessa forma é mais demorada além de sujeita a erros durante o processo.

O resultado disso tudo é que equipes, provavelmente as que usam papel irão ficar sem o registro dos usuários e, assim, sem receber o recurso destinado a esse usuário não cadastrado. Essa é uma situação grave que pode acarretar, portanto, na falta de recursos para população localizada longe de grandes centros, muitas vezes as que mais precisam de apoio do estado. Para contornar esse provável problema ainda não foi proposto pelo Ministério da Saúde nenhuma medida, não se fala se haverá relatórios de feedback para a equipe, para o município e para os estados sobre os usuários cadastrados. Nesse ambiente tem-se, então, um grande risco de diminuir ainda mais o financiamento de um sistema já subfinanciado cronicamente, agravando a situação. Assim, isso pode acarretar consequências desastrosas e irreparáveis com impossibilidade de atendimento por falta de medicamentos, insumos, atrasos no pagamento de profissionais, causando perda para o sistema de saúde e para a população.

Embora seja admirável a tentativa de se mudar o financiamento para uma característica mais moderna baseada no número de pessoas que de fato usam o sistema, não foram feitos levantamentos concretos, não foram usados números que baseassem metas concretas para cada estado e município, não foi anunciado como a gestão vai conseguir efetivar o cadastro dos usuários. Na prática, mudou-se a regra do programa de Atenção Básica, mas não se ensinou a ninguém como é a 
nova regra, somado a isso foram colocadas metas improváveis de serem alcançadas, pouco objetivas e de curto prazo.

Além disso, hoje, não se sabe muito bem como os estados estão e onde estão, sabe-se que já não estão bem e que esperam por melhorias para continuarem no sistema já estabelecido. Essa preocupação também foi expressada por uma carta assinada pela Associação Brasileira de Saúde Coletiva e outras entidades de grupo chamado de "Movimento da Reforma Sanitária", direcionando a carta ao Ministro da Saúde Henrique Mandetta em 30 de outubro de 2019 (MRS, 2019).

O governo anuncia que o programa irá disponibilizar 2 bilhões a mais para a Atenção Primária (BRASIL, 2019a), porém diz isso somente pela metade, não fala em nenhum documento oficial de onde vem o dinheiro, fazendo parecer mais uma promessa do que um fato concreto que se tem o dinheiro. Existe a tese que possa ser um dinheiro remanejado de outros setores da saúde, retirando de um local para colocar em outro, criando certamente problemas onde se retirou o dinheiro. As problemáticas expostas nesse trabalho trazem, portanto, preocupações para os profissionais que trabalham na Atenção Primária e para os usuários desses serviços, necessitando serem discutidas para que se possa pensar em soluções para que não se chegue a consequências maléficas para a população usuária do SUS.

\section{CONCLUSÃO}

Para que a Atenção Primária do Brasil continue a funcionar e para permanecer sendo um sistema de prevenção, cuidado e promoção de saúde é preciso que se viabilize sua existência com recursos financeiros mínimos para tanto. Ter um sistema de saúde eficaz e eficiente, significa diminuir as filas nos sistemas de saúde especializados e com tecnologias mais caras, diminuindo custos e melhorando assim a saúde para todos.

A opção de mudar o sistema é admirável, pois modernizou-se a forma de repasse e de controle, mas não foi feito de forma dialogada como manda a 
constituição e não foi feita de forma responsável delimitando no mundo real o que precisa ser feito. Essa situação tem consequências a médio e longo prazo e precisa de considerações urgentes caso o programa ainda continue em funcionamento, caso contrário os usuários e o SUS irão perder muito com o declínio na efetividade do sistema por falta de recursos para que ele funcione.

Portanto, ainda é necessário o ajuste do projeto Previne Brasil, delimitando com mais clareza o que os gestores devem fazer para terem seus sistemas adequados às novas regras propostas, quais caminhos devem seguir, o que devem fazer, onde pedir ajuda, a quem recorrer. É necessário para isso algumas medidas, como a contratação de profissionais para fazer o cadastro por um curto período de tempo ou pensar em estratégias que façam o cadastro realmente viável. Além disso, é necessário a capacitação dos profissionais de saúde, e dos gestores de saúde para se adequarem às novas regras. Programas de governo podem ser mais bem explicitados, por exemplo, através de cursos online para profissionais e para gestores de saúde municipais, mostrando o caminho a ser trilhado para que se possa atingir o objetivo do programa. 


\section{REFERÊNCIAS BIBLIOGRÁFICAS}

ABRASCO, A. B. DE S. C. Em defesa da atenção primária e do direito universal à saúde? pela revogação da Portaria n 2979 / 19 do Ministério da Saúde. 2019.

BRASIL. Constituição Brasileira. Diário da República, 1a série - no 116, 1988.

BRASIL, MINISTÉRIO DA SAÚDE. PORTARIA No 3.947, DE 28 DE DEZEMBRO DE 2017.

BRASIL, MINISTÉRIO DA SAÚDE. Portaria No 2.979, de 12 de novembro de 2019. 2019a. Disponível em: <http://www.in.gov.br/en/web/dou/-/portaria-n-2.979-de-12-de-novembro-de2019-227652180>. Acesso em: 24 fev. 2020.

BRASIL, MINISTÉRIO DA SAÚDE. RESUMO EXECUTIVO DA 9a REUNIÃO ORDINÁRIA DA COMISSÃO INTERGESTORES TRIPARTITE 31 DE OUTUBRO DE 2019. PARTICIPANTES DO PLENÁRIO DA TRIPARTITE. 2019b.

BRASIL, Presidência da República. Lei Orgânica da Saúde 8080/90. Diário da República, 1a série - no 116, 1990.

CAVALCANTI, P. C. DA S.; NETO, A. V. DE O.; SOUSA, M. F. DE. Quais são os desafios para a qualificação da Atenção Básica na visão dos gestores municipais? SAÚDE DEBATE, v. 39, 2015.

MRS, MOVIMENTO DA REFORMA SANITÁRIA. Carta ao Exmo. Sr. Ministro de Estado da Saúde. Brasília, 2019.

ORGANIZATION, W. H. The World Health Report HEALTH SYSTEMS FINANCING. v. 1, n. Health systems financing, 2010.

ORTIZ-OSPINA, E.; ROSER, M. Financing Healthcare. Our World in Data, 16 jun. 2020. PALUMBI, S. R. Further ANNUAL REVIEWSAnnu. Rev. Ecol. Syst. Disponível em: $<w w w$.annualreviews.org>. Acesso em: 25 fev. 2020.

PROGRAM, P. FOR A N. H. Health Care Systems - Four Basic Models. 2020. Disponível em: <https://www.pnhp.org/single_payer_resources/health_care_systems_four_basic_models.php>. Acesso em: 24 fev. 2020. 